\title{
Influence of kaolinite clay on the chloride diffusion property of cement-based materials
}

\author{
Yingfang Fan ${ }^{\text {a,* }}$, Shiyi Zhang ${ }^{\text {, }}$, Shiho Kawashima ${ }^{\text {, }}$, Surendra P Shah ${ }^{\text {b }}$ \\ a Institute of Road and Bridge Engineering, Dalian Maritime University, Dalian, Liaoning 116026, China \\ ${ }^{\mathrm{b}}$ Civil and Environmental Engineering, Northwestern University, 2145 Sheridan Road, Evanston, IL 60208, USA
}

\section{A R T I C L E I N F O}

\section{Article history:}

Received 3 September 2012

Received in revised form 23 August 2013

Accepted 22 September 2013

Available online 2 October 2013

\section{Keywords:}

Kaolinite clay

Cement-based material

Workability

Strength

Chloride

Permeability

SEM

RCM

\begin{abstract}
A B S T R A C T
To constitute blended cement concrete with high chloride diffusion resistivity, the effects of kaolinite clay on the mechanical properties and chloride diffusivity of cement paste, mortar and concrete were investigated. Ordinary Portland cement was partially replaced by kaolinite clay at $0 \%, 1 \%, 3 \%, 5 \%, 7 \%$ and $9 \%$ by weight of cement. All blended cement-based samples were prepared using a w/c ratio of 0.5 . The microstructure, workability, early-age and long-term flexural strength of pastes were tested. The chloride diffusivity of mortars was measured. And the compressive strength and chloride diffusivity of concrete were measured. Mercury Intrusion Porosimetry (MIP) was employed to evaluate porosity characteristics. Scanning Electron Microscopy (SEM) and Energy Dispersive Spectra (EDS) were applied to investigate the micro morphology and chemical element distribution inside the cement matrix, and the rapid chloride migration (RCM) method was applied to test chloride diffusivity. The MIP test results show that the addition of clay improves the micro-pore structure in the cement paste and limits the introduction of chloride ions. SEM imaging suggests that the kaolinite clay is acting as both filler and accelerator of cement hydration. It is found that the addition of clay alters the water requirement of normal consistency and the setting time of cement, whereas it has little influence on the soundness. Compared to the control, the flexural strength of cement paste with $1 \%$ kaolinite clay increased by $30.41 \%, 39.04 \%, 36.27 \%$ and $38.32 \%$ at $1,3,7$ and 90 days, respectively. The 28-day flexural strength only increased slightly. It is observed that the cement mortar with clay has lower chloride diffusion coefficient values compared to the plain mortar, the 28-day diffusion coefficient of chloride ion $\left(D_{C l}^{-}\right)$of cement mortar is decreased by $53.03 \%$ with $5 \%$ clay. The increase in compressive strength of the cement concrete with clay is $12 \%$, $13.5 \%$, and $28.4 \%$ compared to the control at $1 \%, 3 \%$ and $5 \%$ addition, respectively. The chloride diffusion coefficient of cement concrete decreases exponentially with the clay addition. The reduction of chloride diffusion coefficient of cement concrete is $8.68 \%$ and $18.87 \%$ at $1 \%$ and $5 \%$ clay, respectively. The 28 -day compressive strength increases linearly with the chloride diffusion coefficient of the concrete.
\end{abstract}

(c) 2013 Elsevier Ltd. All rights reserved.

\section{Introduction}

It is well known that concrete is a porous material, which makes it susceptible to attack by chloride ions in seawater and deicing environments. Penetration of chloride ions through the connected pore network promotes corrosion of steel reinforcement, potentially leading to early deterioration of the structure. During the past decades, many concrete structures in service within chloride environments (e.g. seawater, deicing salt, chemical plant, etc.) were damaged much earlier than their target service lives. Developing construction materials with high chloride penetration resistance would be an efficient and fundamental way to extend

\footnotetext{
* Corresponding author. Tel.: +86 (0) 41184729610.

E-mail address: fanyf72@yahoo.com.cn (Y. Fan).
}

the service life, and subsequently increase the sustainability of concrete structures.

Chloride diffusivity through concrete and cement paste is influenced by many factors, including water cement ratio, the type of cement and other mix constituents, concrete mix proportions, compaction and curing condition. To reduce the chloride permeability of concrete in practice, various types of plasticizers are used, but the strength or ductility is not always satisfactory. With the application of nanotechnology in civil engineering, the incorporation of nanoparticles in cement-based materials has been demonstrated to improve the properties of concrete. In previous studies, additives such as nano $\mathrm{SiO}_{2}$, nano $\mathrm{TiO}_{2}, \mathrm{Fe}_{2} \mathrm{O}_{3}, \mathrm{Al}_{2} \mathrm{O}_{3}$, and clays were found to improve material strength and fatigue characteristics while enhancing ductility and other durability properties of engineering materials [1-4]. However, there are only a limited number of studies on the use of nanoparticles to enhance the 
durability of cement-based materials. It is reported that for cement mortars with nanoparticles $\left(\mathrm{Fe}_{2} \mathrm{O}_{3}, \mathrm{Al}_{2} \mathrm{O}_{3}, \mathrm{TiO}_{2}\right.$, and $\left.\mathrm{SiO}_{2}\right)$ and nanomontmorillonite at $1 \%$ by weight of cement, the reduction of 28-day $\mathrm{D}_{\mathrm{Cl}}{ }^{-}$was in the order of Clay (montmorillonite) $>\mathrm{SiO}_{2}>$ $\mathrm{TiO}_{2}>\mathrm{Al}_{2} \mathrm{O}_{3}>\mathrm{Fe}_{2} \mathrm{O}_{3}$ [5]. Considering the low cost of clay, the use of clay in concrete to reduce chloride permeability seems to be promising. Since the two-layer structure of kaolinite clay resists water molecules from going through the sheets, the addition of clay in concrete can take an active effect on lowering the permeability of cement mortar [6,7]. However, the early age and long term properties of the cement concrete with clay are still unclear and needs further investigation. To realize the application of clay-modified cement-based materials in practical engineering, it is necessary to explore their mechanical and durability behavior.

The objective of this research is to investigate the effect of kaolinite clay on the workability, strength and chloride diffusion coefficient of cement-based mixtures (i.e. cement, mortar, and concrete). The effects of addition amount and dispersion method on the porosity characteristics of cement paste are studied. The porosity and microstructure are evaluated through Mercury Intrusion Porosimetry (MIP) and Scanning Electron Microscope (SEM) and Energy Dispersive Spectra (EDS) methods.

\section{Materials and methods}

\subsection{Material properties}

Ordinary Portland cement of Type 42.5R was used in all mixes. A commercially available kaolinite clay powder was used in this study. The kaolinite clay has a crystalline structure and contains silicon. The relative density, average size, and $\mathrm{pH}$ value of the clay is $2.58,370$ and $7.9 \mathrm{~nm}$. Its theoretical formula is $\mathrm{Al}_{2} \mathrm{Si}_{2} \mathrm{O}_{5}(\mathrm{OH})_{4}$ [8]. The chemical composition of the cement and clay are listed in Table 1. To clarify its microstructure, X-ray diffraction (XRD) analysis and SEM/EDS techniques were carried out on the neat clay powder. The resulting XRD and SEM/EDS images of the clay are shown in Fig. 1. From the EDS spectra shown in Fig. $1 \mathrm{~b}$.

Table 1

Chemical composition of PO42.5R cement and clay (wt.\%).

\begin{tabular}{lll}
\hline Compound (\%) & Cement & Clay \\
\hline $\mathrm{CaO}$ & 59.30 & 0.28 \\
$\mathrm{SiO}_{2}$ & 21.91 & 47.80 \\
$\mathrm{Al}_{2} \mathrm{O}_{3}$ & 6.27 & 41.80 \\
$\mathrm{Fe}_{2} \mathrm{O}_{3}$ & 3.78 & 0.30 \\
$\mathrm{MgO}$ & 1.64 & 0.03 \\
$\mathrm{SO}_{3}$ & 2.41 & - \\
$\mathrm{K}_{2} \mathrm{O}$ & - & 0.58 \\
$\mathrm{TiO}_{2}$ & - & 0.02 \\
$\mathrm{Na}_{2} \mathrm{O}$ & - & 0.06 \\
\hline
\end{tabular}

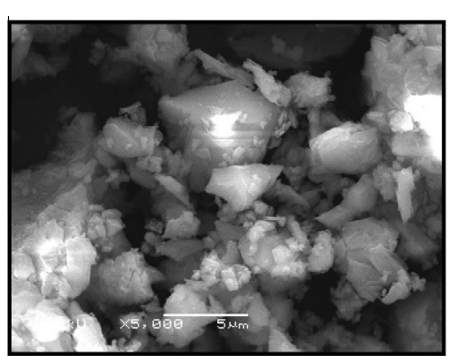

(a)

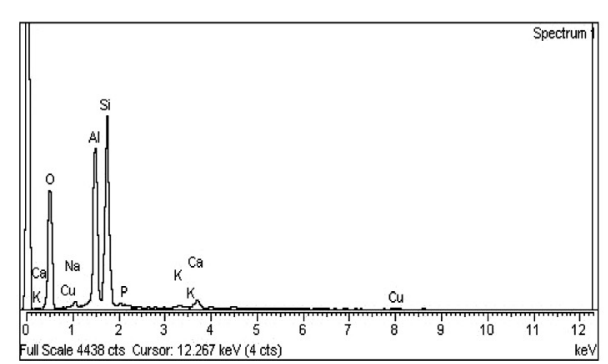

(b)

\subsection{Specimen fabrication}

To prepare the paste samples, $1 \%, 3 \%, 5 \%, 7 \%$ and $9 \%$ of cement by weight was replaced by kaolinite clay. An effective water-tobinder ratio of 0.5 was maintained in all mixes. ISO standard sand was applied. For mortar, the cement-sand ratio was $1: 3$ by weight. For concrete, the cement: water: sand: aggregate ratio was 350:175:619:1256.

To achieve a good dispersion of the clay in pastes and mortars, the clay was first dispersed in water in a rotary mixer at high speed for 5 mins. The mixing protocols for cement paste and mortar are listed in Table 2, respectively. In the case of concrete, the clay was first dispersed in water by an ultrasonic dispersion method. Then, the dispersed clays were mixed with fine and coarse aggregate.

For paste, the mix is poured into oiled molds to form prisms of size $40 \mathrm{~mm} \times 40 \mathrm{~mm} \times 160 \mathrm{~mm}$, to be used for compressive strength testing. For mortar and concrete, the mix is poured into oiled molds to form cubes of size $150 \mathrm{~mm} \times 150 \mathrm{~mm} \times 150 \mathrm{~mm}$ and cylinders of size $\Phi 100 \mathrm{~mm} \times 50 \mathrm{~mm}$ to be used for compressive strength and permeability testing, respectively. The fabricated samples were demolded after $24 \mathrm{~h}$ and then cured in the standard curing condition (at a temperature of $20 \pm 3{ }^{\circ} \mathrm{C}$ and a relative humidity of $95 \%$ ) until the prescribed period. For all mixes at each age, three specimens were fabricated and tested to ensure the statistical reliability of test results.

\subsection{Methods}

\subsubsection{Mercury Intrusion Porosimetry (MIP)}

The chloride resistance of cement and concrete is highly dependent on the porosity of the material in terms of pore size, pore distribution and interconnectivity of the pore system [9]. The inclusion of nanoparticles will lead to a change in the pore size distribution of concrete. Therefore it is necessary to understand the influence of different nanoparticle additions on the porosity characteristics of the cement matrix. The water vapor adsorption test, nitrogen adsorption test, and MIP test are the most popular methods to examine the porosity characteristics of cement-based material $[10,11]$.

In this study, the MIP test was implemented to investigate the effect of kaolinite clay on the pore size distribution of cement pastes. The porosity of hardened cement paste was measured using high-pressure porosimeter Micrometrics Auto-Pore II9200 (with pressure range up to $400 \mathrm{MPa}$ ).

\subsubsection{Scanning Electron Microscopy (SEM)}

SEM is a well-established method that can offer useful information concerning the structure of material [12]. To better understand the evolution of the microstructure of cement paste samples with clay addition, they were observed by a JSM-6360LV

Fig. 1. SEM/EDS and XRD spectra of neat clay powder. (a) SEM micrograph. (b) EDS result. (c) XRD result. 
Table 2

Mix protocol for cement compositions and cement mortar.

\begin{tabular}{|c|c|c|}
\hline \multirow[t]{2}{*}{ Time } & \multicolumn{2}{|l|}{ Procedure } \\
\hline & Cement & Cement mortar \\
\hline $0: 00$ & Disperse the clay in water & Disperse the clay in water \\
\hline 15:00 & Add dry ingredients, mixing on low speed (140 rpm) & Add dry cement ingredients, mixing on low speed \\
\hline $17: 15$ & & Add sand in $30 \mathrm{~s}$, mixing on low speed \\
\hline 20:00 & Stop mixing, scrap edges of mixer & Stop mixing, scrap edges of mixer \\
\hline 21:00 & Mix on high speed (285 rpm) & Mix on high speed \\
\hline 26:00 & End of mixing & End of mixing \\
\hline
\end{tabular}

SEM system. The applied accelerating voltage is $20 \mathrm{kV}$. The EDS technique was applied to analyze the elemental distribution in the samples, as well. The EDS plots provide the presence of chemical elements, which will confirm the composition of crystal in the concrete samples. Small fractured samples or powder samples were coated gold with the thickness of 5 to $10 \mathrm{~nm}$ to make it conductive.

\subsubsection{Testing the chloride diffusion property of concrete}

To evaluate the chloride permeability of cement-based materials, a series of testing methods (e.g. the AASHTO T259 test, Bulk diffusion test (NordTestNTBuild 443), AASHTO T277 (ASTM C1202) test, electrical migration techniques, the rapid migration test [13-18], resistivity techniques, pressure penetration techniques, etc.) have been developed. Rapid chloride migration (RCM) method proposed by Tang and Nilsson [17] was applied in this study. The principle of this method is to generate chloride penetration through the sample by a solution concentration gradient and accelerate the movement of chloride using an electrical field.

A migration cell was set up with a specimen $50 \mathrm{~mm}$ thick and $100 \mathrm{~mm}$ in diameter. The testing setup used in this paper is shown in Fig. 2. The relationship between the applied initial current and the testing time is shown in Table 3. After a specified duration (depending on the applied current), the specimens were removed and split. Then, a $0.1 \mathrm{~N}$ silver nitrate solution was sprayed onto the surface of the split samples. If chloride rises up to the surface after spraying the solution on the surface for 15 mins, it will create a whitish color. Otherwise it will become brown. The color change border indicates the chloride penetration depth. The depth of the chloride penetration was measured. Then the depth was used to determine the diffusion coefficient through the Nernst-Einstein equation, which is described as follows $[19,20]$,
$D_{R C M}=2.872 \times 10^{-6} \frac{T h\left(x_{d}-a \sqrt{x_{d}}\right)}{t}$

$a=3.338 \times 10^{-3} \sqrt{T h}$

where $D_{R C M}$ is the chloride diffusion coefficient tested by the RCM method, $\left(\mathrm{m}^{2} / \mathrm{s}\right) ; T$ is the average temperature of the initial and final temperature of the anode solution, $(K) ; h$ is the height of the testing specimen, $(m) ; x_{d}$ is the diffusion depth of chloride anion, $(m) ; t$ is the electricity test time, $(s) ; \alpha$ is a dimensionless constant.

\section{Results and discussions}

\subsection{Effect of clay on the microstructure of cement paste}

The test samples for microstructures were obtained from the central part of cement specimens. Fig. 3 shows the SEM micrographs of cement paste with clay additions.

From the SEM images of the clay-modified cement paste samples, plate structures become visible once the clay addition is increased to $1.5 \mathrm{wt} . \%$. These are apparent at 0.75 and $1.0 \mathrm{wt}$.\% additions. The SEM observations suggest that the kaolinite clay is acting as filler, where the porosity in cement was improved once the clay was well-dispersed in the cement paste [21]. It can be derived that, being a pozzolanic material [22,23], kaolinite clay increase the chemical reactions in the paste.

\subsection{Effect of clay on the pore character of cement paste}

From the MIP test, the pore size distribution parameters such as total specific pore volume, most probable pore diameter, pore size distribution, porosity, average diameter, and median diameter (volume) are obtained and listed in Table 4. The developments of

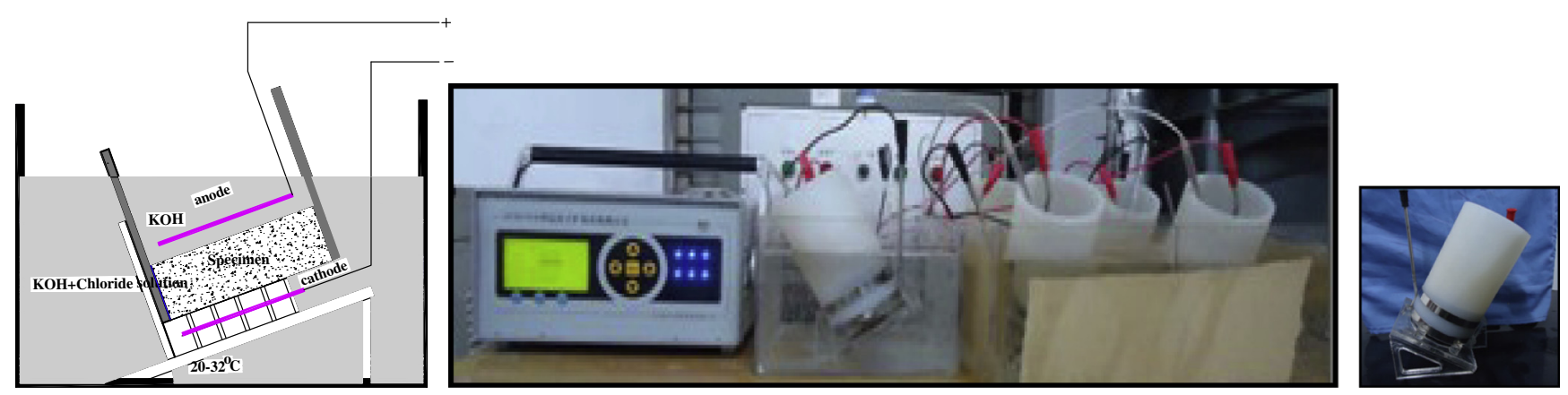

Fig. 2. Rapid chloride migration test setup used in this paper. (a) Schematic. (b) Actual. (c) Silica tube.

Table 3

Initial current and testing time.

\begin{tabular}{|c|c|c|c|c|c|c|}
\hline Initial current $\mathrm{I}_{0} / \mathrm{mA}$ & $\mathrm{I}_{0}<5$ & $5 \leqslant \mathrm{I}_{0}<10$ & $10 \leqslant \mathrm{I}_{0}<30$ & $30 \leqslant \mathrm{I}_{0}<60$ & $60 \leqslant \mathrm{I}_{0}<120$ & $120 \leqslant \mathrm{I}_{0}$ \\
\hline Testing time/h & 168 & 96 & 48 & 24 & 8 & 4 \\
\hline
\end{tabular}



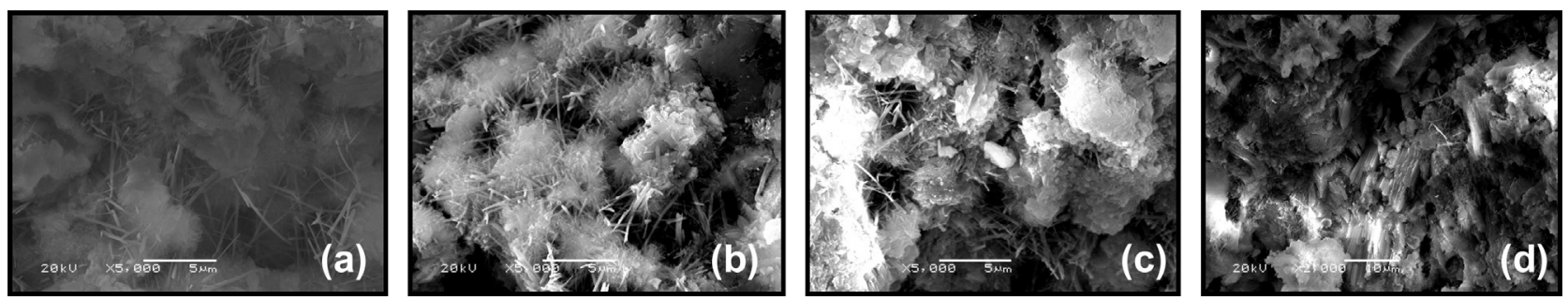

Fig. 3. SEM micrograph of control and clay-modified cement paste. (a) Without clay. (b) 0.75 wt.\% clay addition. (c) 1.0 wt.\% clay addition. (d) 1.5 wt.\% clay addition.

Table 4

Total specific pore volumes and most probable diameters of cement.

\begin{tabular}{|c|c|c|c|c|c|}
\hline Series & Total specific pore volume $(\mathrm{mL} / \mathrm{g})$ & Mode pore diameter $(\mathrm{nm})$ & Porosity (\%) & Average pore diameter (nm) & Medium pore diameter $(\mathrm{nm})$ \\
\hline Plain & $0.0031(0.00)$ & $349.92(0.00)$ & $28.42(0.00)$ & $40.7(0.00)$ & $121.6(0.00)$ \\
\hline NCM1 & $0.0035(12.90)$ & $151.04(-56.83)$ & $26.47(-6.86)$ & $35.7(-12.29)$ & $92.9(-23.60)$ \\
\hline NCM2 & $0.0024(-22.58)$ & $151.01(-56.84)$ & $23.17(-18.48)$ & $32.3(-20.64)$ & $69.0(-43.26)$ \\
\hline
\end{tabular}

pore size distribution and porosity ratio inside the concrete samples are examined. Differential curves of pore size distribution of the controlled and modified concretes are obtained, shown in Fig. 4.

The results show that the clay will improve the pore size distribution in the cement paste. With $1 \%$ of clay addition, the porosity in the cement paste has a reduction of $18.48 \%$, whereas the average, medium and mode pore diameter have a reduction of $20.64 \%, 43.26 \%$ and $56.84 \%$, respectively. And this effect increases with addition level, as shown in Fig. 5, which shows the relationship between the pore size distribution parameters (porosity, average pore diameter, total pore area, total intrusion volume) and clay addition.

\subsection{Effect of clay on workability of cement paste}

To understand the workability of the clay modified cement material, flow ability, water requirement of normal consistency, setting time and soundness were examined in this paper.

\subsubsection{Flow ability and water requirement of normal consistency}

The control plain paste and five cement pastes with clay additions of $1 \%, 3 \%, 5 \%, 7 \%$ and $9 \%$ were tested to determine the flowability and water requirement of normal consistency. The test

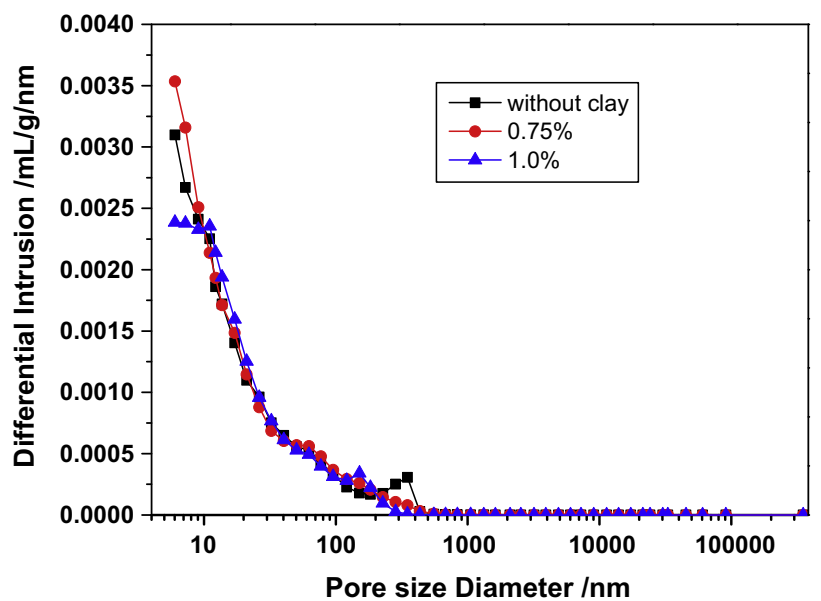

(a)

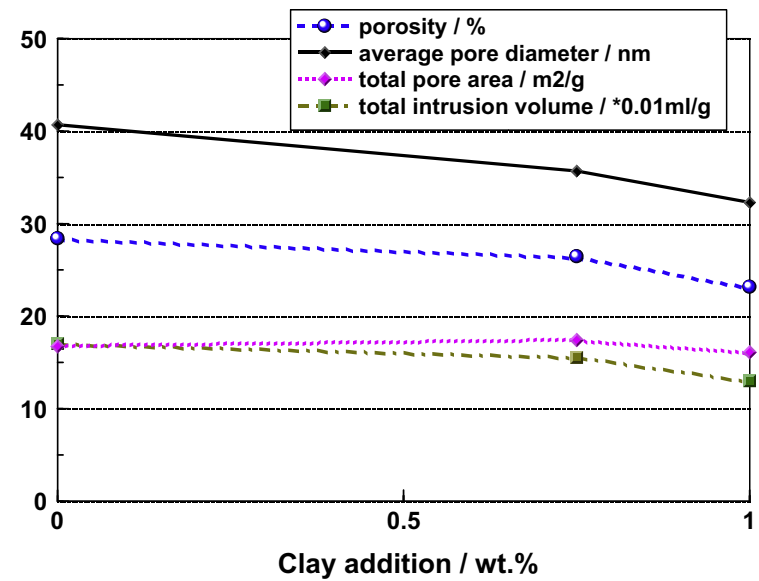

Fig. 5. Relation between the addition of clay and pore character of control and modified cement paste.

procedure complies with the present code 'Test methods of cement and concrete for highway engineering' (JTG E30-2005). The results are shown in Table 5 .

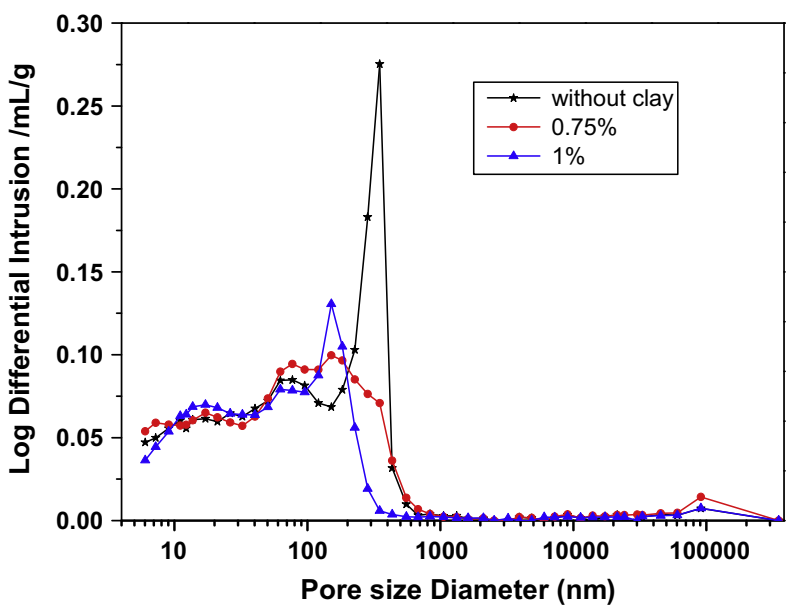

(b)

Fig. 4. Cumulative pore diameter distribution. (a) Cumulative pore diameter distribution, (b) Differential pore diameter distribution. 
Table 5

Fluidity and water demand of normal consistency of cement paste.

\begin{tabular}{llll}
\hline Series & $\begin{array}{l}\text { Clay addition } \\
\text { (wt.\%) }\end{array}$ & $\begin{array}{l}\text { Flow ability } \\
(\mathrm{cm})\end{array}$ & $\begin{array}{l}\text { Water demand of normal } \\
\text { consistency }(\mathrm{kg})\end{array}$ \\
\hline Control & 0 & 18.70 & 133.25 \\
CM1 & 1 & 16.83 & 147.11 \\
CM2 & 3 & 16.25 & 149.07 \\
CM3 & 5 & 15.68 & 154.11 \\
CM4 & 7 & 15.13 & 157.85 \\
CM5 & 9 & 14.30 & 162.07 \\
\hline
\end{tabular}

Table 6

Setting time of the cement pastes with clay additions.

\begin{tabular}{lllllll}
\hline Clay additions (wt.\%) & 0 & 1 & 3 & 5 & 7 & 9 \\
\hline Initial setting time/min & 190 & 185 & 170 & 175 & 170 & 170 \\
Final setting time/min & 290 & 280 & 275 & 265 & 270 & 280 \\
\hline
\end{tabular}

The results show that the addition of clay can reduce the flowability of cement and increase the water demand of normal consistency. The greater the clay addition, the greater the effect on the flowability and the water demand of normal consistency. The decrease in flowability and increase in water demand is likely due to the higher water adsorption and increased exhibited by clays [1]. The relationship between clay addition and the reduction ratio of flowability is close to linear. A linear relationship exists between clay addition and the ratio of increase in water demand of normal consistency, as well.

\subsubsection{Setting time}

The setting time of the cement pastes with clay additions of $0 \%$, $1 \%, 3 \%, 5 \%, 7 \%$ and $9 \%$ were measured by a Vicat apparatus. The initial setting time can be defined as "time taken by paste to stiffen to such an extent that the Vicatt's needle is not permitted to move down through the paste through $25 \mathrm{~mm}$." The final setting time can be defined as "it is the time after which the paste becomes so hard that Vicatt's $5 \mathrm{~mm}$ needle does not sinks visibly and leave no impression". The experiments were executed according to the present standard JTGE30-2005(The methods of cement and concrete for highway engineering) [24].

The initial and final setting time of each paste is given in Table 6. The relation between the depth of penetration and the setting time is plotted in Fig. 6.

The results show that the addition of clay has little effect on both the initial and final setting time of the cement. Generally, both the initial and final setting time of the cement paste with clay addition will have a slight decrease.

\subsubsection{Soundness}

The soundness of the cement pastes with clay additions of $0 \%$, $1 \%, 3 \%, 5 \%, 7 \%$ and $9 \%$ were examined. The experiments were executed according to the present standard JTGE30-2005 (The methods of cement and concrete for highway engineering) [24].

For the control plain cement sample and cement samples with five various clay additions, no cracking and wrapping occurred on the samples. Therefore, it is shown that the addition of clay does not affect the soundness of cement paste.

\subsection{Effect of clay on strength of cement paste and concrete}

\subsubsection{Bending strength of cement paste}

After being cured in the standard curing condition for $1,3,7,14$, 28 and 90 days, flexural tests were performed. The present Chinese standard JTG E30-2005 (Test methods of cement and concrete for highway engineering, China) was applied. The bending strength of the cement pastes with clay additions of $0,1,3$ and $5 \mathrm{wt} . \%$ are obtained, and the results are shown in Table 7. Development of strength with curing age for the cement paste at various clay additions are compared and discussed. A summary of the flexural strength increase ratio (SIR) for each mix is shown in Fig. 7. The value of FSIR is calculated as:

Flexural Strength Increase Ratio $=($ Flexural strength of modified sample - Flexural strength of control sample)/Flexural strength of control sample $\times 100 \%$.

Early-age flexural strength gain

Fig. 8 shows the developing tendency of the flexural strength of cement paste with various clay additives at the early age.

It is apparent that the addition of clay can increase the flexural strength of the cement paste. When kaolinite clay was mixed into fresh cement paste at $1 \%$ by weight of cement, the value of flexural

Table 7

The bending testing result

\begin{tabular}{lllll}
\hline \multirow{2}{*}{ Curing age/days } & \multicolumn{4}{l}{ Flexural strength/MPa (FSIR/\%) } \\
& $0 \%$ & $1 \%$ & $3 \%$ & $5 \%$ \\
\hline 1 & $1.94(0.00)$ & $2.53(30.41)$ & $2.36(21.65)$ & $2.78(43.30)$ \\
3 & $4.56(0.00)$ & $6.34(39.04)$ & $5.17(13.38)$ & $5.79(26.97)$ \\
7 & $6.01(0.00)$ & $8.19(36.27)$ & $6.94(15.47)$ & $7.66(27.45)$ \\
14 & $6.19(0.00)$ & $7.33(18.42)$ & $9.21(48.79)$ & $9.22(48.95)$ \\
28 & $7.48(0.00)$ & $7.58(1.34)$ & $7.89(5.48)$ & $7.28(-2.67)$ \\
90 & $7.75(0.00)$ & $10.72(38.32)$ & $10.17(31.23)$ & $9.01(16.26)$
\end{tabular}

Note. The number in the () is FSIR.

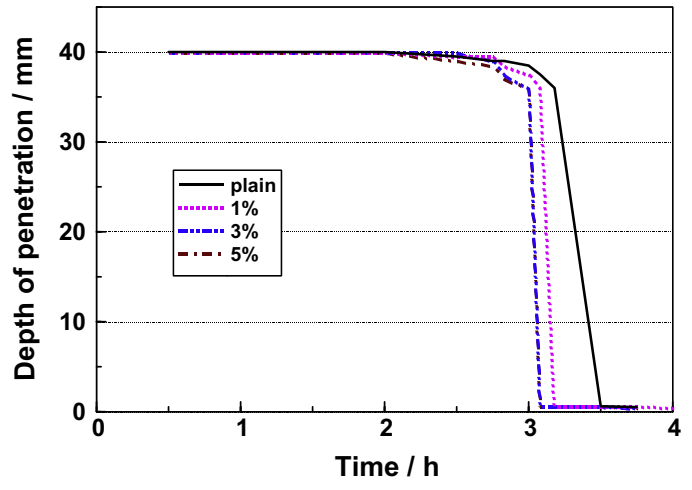

(a)

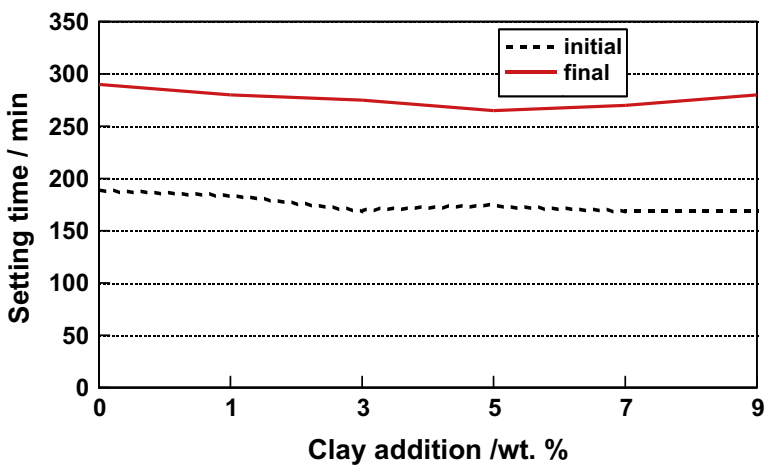

(b)

Fig. 6. Setting time of the cement pastes. (a) Development of the penetration depth with time. (b) Relation between the setting time and clay additions. 


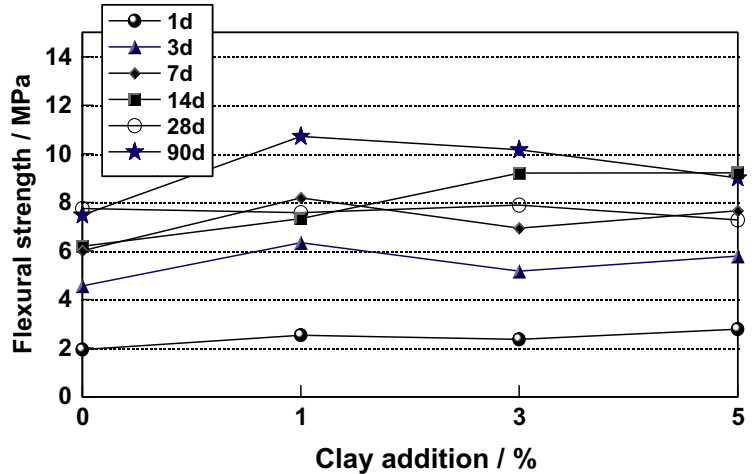

(a)

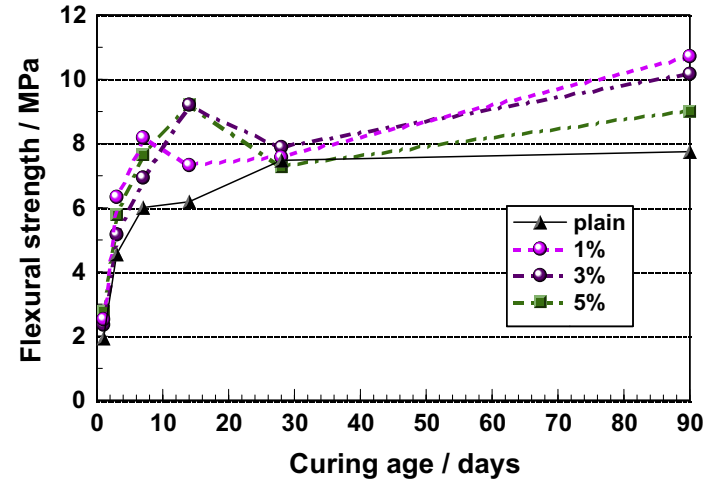

(b)

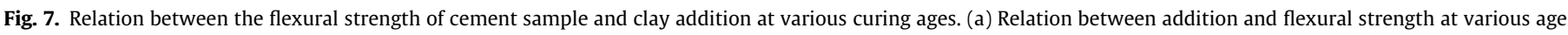
(b) Relation between curing age and flexural strength at various additions.

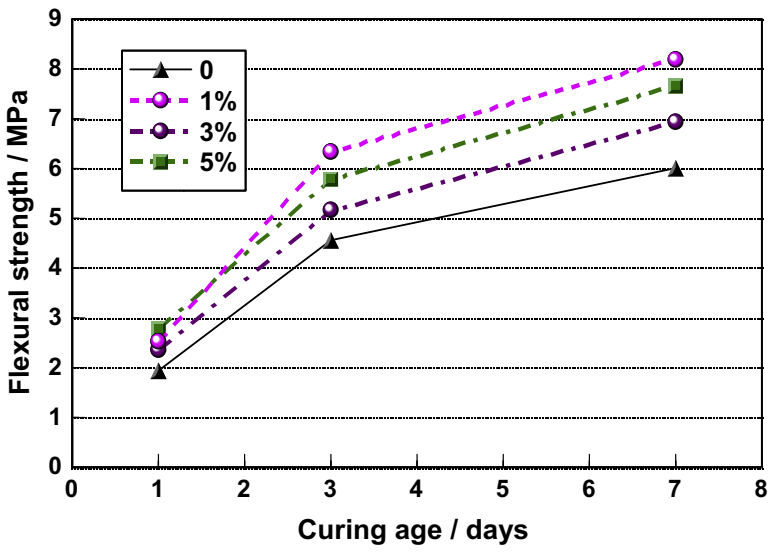

Fig. 8. Flexural strength of cement paste at early age.

strength was increased by $30.41 \%, 39.04 \%$, and $36.27 \%$ at 1,3 and 7 days, respectively. The increase in strength induced by the clay was in the order of Cement $+1 \%$ clay $>$ Cement $+5 \%$ clay $>$ Cement $+3 \%$ clay $>$ Control cement.

Long-term flexural strength

Fig. 11 shows the evolution of flexural strength of cement paste at later age. It is apparent that the addition of clay can increase the flexural strength of the cement paste at 90 days. When kaolinite

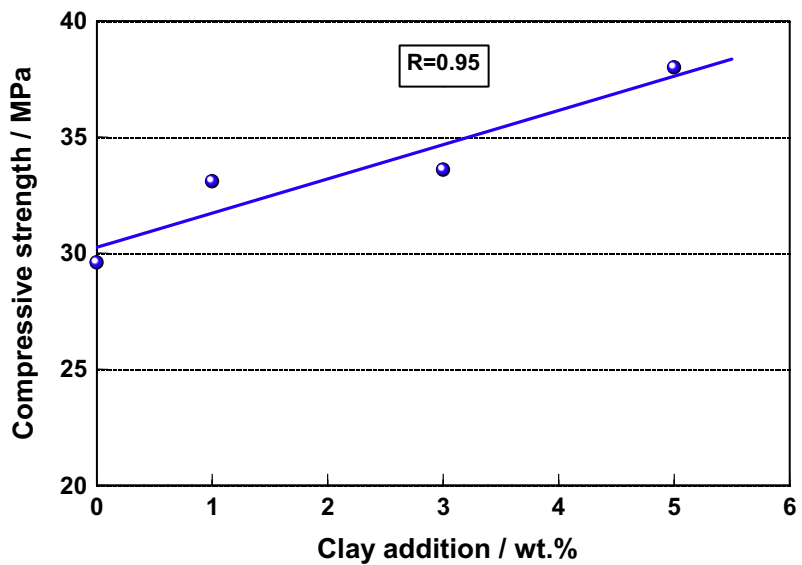

Fig. 9. Relation between the compressive strength of concrete and clay addition. clay was mixed into fresh cement paste at $1 \%$ and $3 \%$ by weight of cement, the value of flexural strength was increased by $38.32 \%$ and $31.23 \%$, respectively (shown in Table 9). The increase in $90-$ day flexural strength induced by the clay was in the order of Cement $+1 \%$ clay $>$ Cement $+3 \%$ clay $>$ Cement $+5 \%$ clay $>$ Control cement.

\subsubsection{Compressive strength of modified concrete}

The compressive tests were performed on concrete specimens at 28 days. Three samples per batch were tested on an electrohydraulic servo compressive testing system (YAW-YAW2000A) with a capacity of $2000 \mathrm{kN}$. The test procedure complies with the present Chinese standard GB/T 50081-2002 (Standard for test method of mechanical properties on ordinary concrete, China) [25]. The loading rate on the cubes was $0.5 \mathrm{MPa} / \mathrm{s}$. The development of compressive strength with clay addition is shown in Fig. 9.

When kaolinite clay was mixed into fresh cement concrete at $1 \%, 3 \%$ and $5 \%$ by weight of cement, the increase in compressive strength was $12 \%, 13.5 \%$, and $28.4 \%$ above the control value, respectively. From Fig. 9, it can be seen that the compressive strength of concrete specimens increases linearly with the clay additions.

\subsection{Effect of clay on permeability of cement-based material}

\subsubsection{Effect of clay on permeability of cement mortar}

Based on the RCM method, chloride penetration depth of cement mortar at 14, 28 and $56 \mathrm{~d}$ curing ages are obtained. Then the chloride diffusion coefficient for conventional cement mortar and cement mortar with various clay additions cured for 14,28 , and 56 days were calculated. A summary of the reduction diffusion coefficient ratio (RDCR) for all mixes are shown in Table 8. The value of RDCR is calculated as.

Reduction Diffusion Coefficient Ratio $=($ Diffusion coefficient of modified sample-Diffusion coefficient of control sample)/Diffusion coefficient of control sample $\times 100 \%$.

Fig. 10 shows the evolution of chloride diffusion coefficient of cement mortar with curing age. It is observed that the modified samples have lower chloride diffusion coefficient values compared to the unmodified control mix. When kaolinite clay was mixed into fresh cement mortar at $1 \%$ by weight of cement, the value of $D_{C l}^{-}$ was decreased by $29.03 \%$ and $20.80 \%$ at 28 and 56 days, respectively. When kaolinite clay was mixed into fresh cement mortar at $5 \%$ by weight of cement, the value of 28 -day $D_{C l}^{-}$was decreased by $53.03 \%$. 


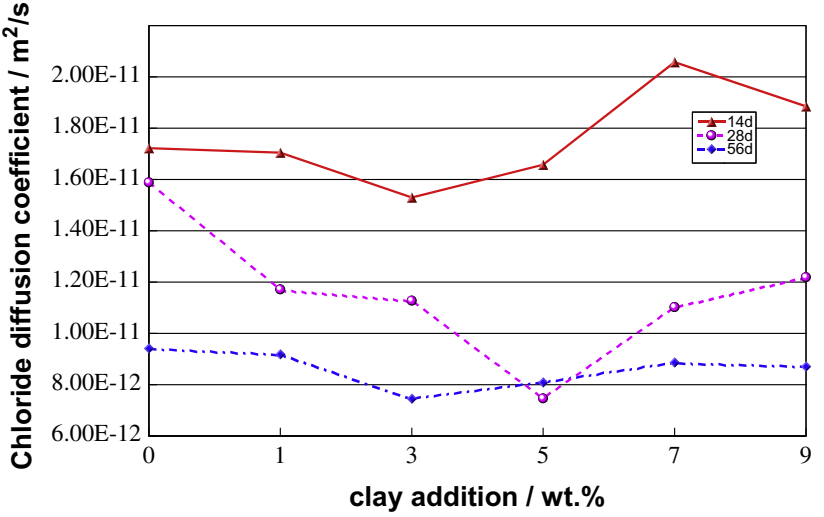

(a)
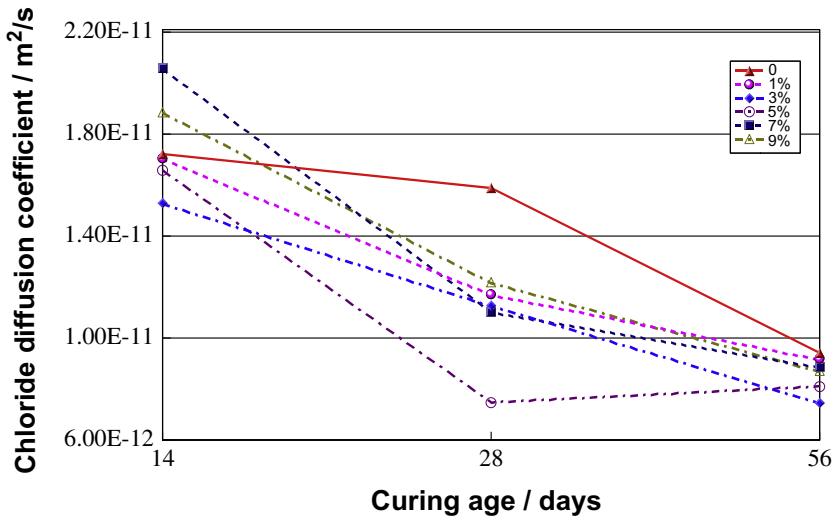

(b)

Fig. 10. The relationship between chloride diffusion coefficient of cement mortar and clay additions.

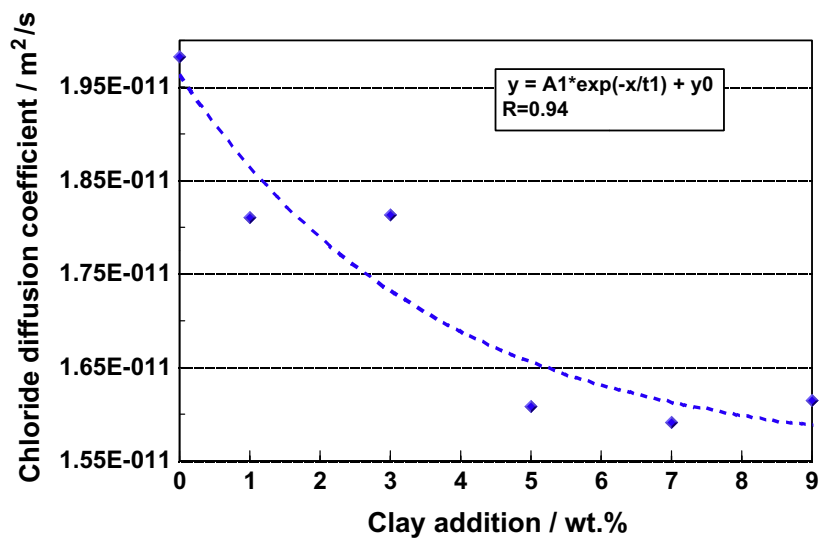

Fig. 11. The relationship between chloride diffusion coefficient of concrete samples and clay additions at 28 ages.

\subsubsection{Effect of clay on chloride permeability of cement concrete}

Based on the RCM method, the chloride permeability tests were performed on concrete specimens with and without clay at 28 ages. The depths of chloride penetration of the tested concrete specimens were measured. Chloride diffusion coefficients of the tested specimens were then calculated by Eq. (1). The development of the chloride diffusion coefficients with clay additions are shown in Fig. 11.

Fig. 11 shows the evolution of chloride diffusion coefficient of concrete at 28 curing ages. It is indicated that the chloride diffusion coefficient of cement concrete decrease exponential with the clay additions. When kaolinite clay was mixed into fresh cement concrete at $1 \%$ and $5 \%$ by weight of cement, the reduction of chloride diffusion coefficient is $8.68 \%$ and $18.87 \%$, respectively. With the

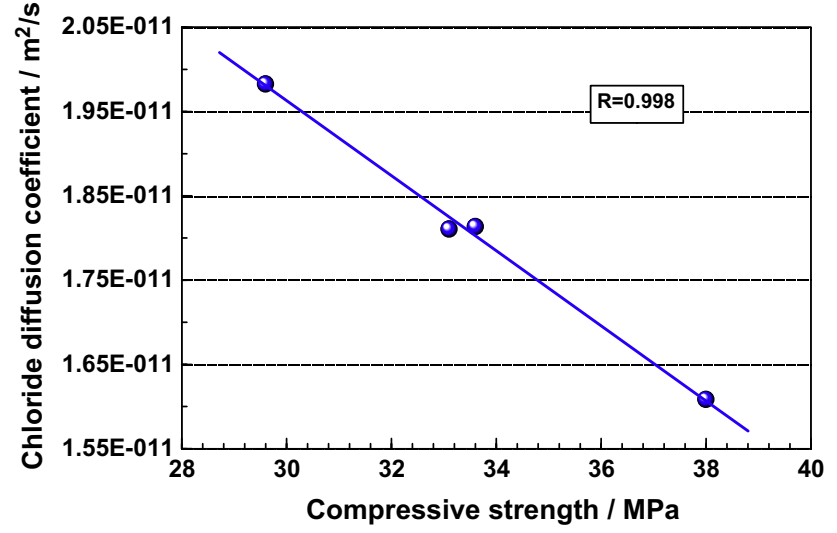

Fig. 12. Relation between compressive strength and chloride diffusion coefficient of cement concrete.

increase of the clay content, the reduction of the chloride diffusion coefficient will slow down.

\subsubsection{Relation between the diffusion property and the compressive strength}

The relation between compressive strength and the chloride diffusion coefficient of the control and clay modified concrete are analyzed, which is shown in Fig. 12.

Fig. 12 shows that a linear relation exists between the 28-day compressive strength and the chloride diffusion coefficient of the clay modified concrete. That is, the 28-day compressive strength increases linearly with the chloride diffusion coefficient of the concrete.

Table 8

Testing results of chloride diffusion coefficient of cement mortar.

\begin{tabular}{|c|c|c|c|c|}
\hline Series & Clay addition (wt.\%) & & Chloride diffusion coefficient $/ \mathrm{m}^{2} / \mathrm{s}$ (RDCR/\%) & \\
\hline & & $14 \mathrm{~d}$ & $28 \mathrm{~d}$ & $56 \mathrm{~d}$ \\
\hline Plain & 0 & $1.72 \mathrm{e}-11(0.00)$ & $1.59 \mathrm{e}-11(0.00)$ & $9.42 \mathrm{e}-12(0.00)$ \\
\hline CC1 & 1 & $1.71 \mathrm{e}-11(-1.01)$ & $1.17 e-11(-26.28)$ & $9.19 \mathrm{e}-12(-2.43)$ \\
\hline $\mathrm{CC} 2$ & 3 & $1.53 \mathrm{e}-11(-11.2)$ & $1.13 e-11(-29.03)$ & $7.46 e-12(-20.80)$ \\
\hline $\mathrm{CC} 3$ & 5 & $1.66 \mathrm{e}-11(-3.74)$ & $7.46 e-12(-53.03)$ & $8.09 \mathrm{e}-12(-14.09)$ \\
\hline CC4 & 7 & $2.06 e-11(19.48)$ & $1.10 \mathrm{e}-11(-30.62)$ & $8.87 e-12(-5.82)$ \\
\hline CC5 & 9 & $1.89 \mathrm{e}-11(9.48)$ & $1.22 \mathrm{e}-11(-23.28)$ & $8.71 \mathrm{e}-12(-7.49)$ \\
\hline
\end{tabular}

Note. The number in the () is RCDR. 


\section{Conclusions}

The potential of incorporating kaolinite clay to enhance the chloride penetration resistivity of concrete structures was investigated. The influences of kaolinite clay on the mechanical and chloride penetration properties of cement-based materials were measured. Based on the results obtained in the present study, the following conclusions can be drawn:

(1) The addition of kaolinite clay improved the micro-pore size distribution in cement paste. The porosity in the cement paste has a reduction of $18.48 \%$, whereas the average, medium and mode pore diameter have a reduction of $20.64 \%$, $43.26 \%$ and $56.84 \%$ at $1 \%$ clay, respectively. Compared with the control cement paste, the average pore diameter of paste with 0.75 and $1.0 \mathrm{wt} . \%$ clay addition achieves a reduction of $6.9 \%$ and $18.5 \%$, respectively; and the reduction of the diameter limit the introduction of the chloride ions. The SEM observations suggest that the kaolinite clay is acting as filler, as well as an accelerator of cement hydration.

(2) The addition of clay increases both early-age and long-term flexural strength of the cement paste, whereas the 28-day flexural strength only has a slight increase. The flexural strength of cement paste with $1 \%$ kaolinite clay is increased by $30.41 \%, 39.04 \%, 36.27 \%$ and $38.32 \%$ at $1,3,7$ and 90 curing ages, respectively. The experimental results show that the addition of clay alters the water requirement of normal consistency and the setting time of the cement paste, whereas it has little effect on the soundness.

(3) It is observed that the cement mortar with clay has lower chloride diffusion coefficient values compared to the control mortar. The 28-day $D_{C l}^{-}$of cement mortar with $1 \%$ clay is decreased by $29.03 \%$ and $20.80 \%$ at 28 and 56 days, respectively. The 28-day $D_{C l}^{-}$of cement mortar is decreased by $53.03 \%$ at $5 \%$ clay

(4) The compressive strength and the chloride diffusion resistivity of the cement concrete with clay are higher than plain cement concrete with the same water to cement ratio. The enhancement of compressive strength of concrete is $12 \%$, $13.5 \%$, and $28.4 \%$ above the control value at $1 \%, 3 \%$ and $5 \%$ clay, respectively. The chloride diffusion coefficient of cement concrete decreases exponentially with the clay additions. The reduction of chloride diffusion coefficient of cement concrete is $8.68 \%$ and $18.87 \%$ at $1 \%$ and $5 \%$ clay, respectively. The 28-day compressive strength increases linearly with the chloride diffusion coefficient of the concrete.

\section{Acknowledgements}

This research was financially supported by the National Natural Science Foundation of China (Grant No. 51178069), National Natural Science Foundation of China (Grant No. 50708010), Program for New Century Excellent Talents in University, Liaoning Provincial
Funded project (Grant No. 20092149), and the Fundamental Research Funds for the Central Universities.

\section{References}

[1] Tregger N, Pakula M, Shah SP. Influence of micro- and nanoclays on fresh state of concrete. Transport Res Rec 2010;2141:68-74.

[2] Goh SW, Akin M, You Z, Shi X. Effect of deicing solutions on the tensile strength of micro- or nano-modified asphalt mixture. Constr Build Mater 2011;25:195-200.

[3] Sanchez F, Sobolev K. Nanotechnology in concrete-a review. Constr Build Mater 2010;24:2060-71.

[4] Zhang M, Li H. Pore structure and chloride permeability of concrete containing nano-particles for pavement. Constr Build Mater 2011;25:608-16.

[5] He X, Shi X. Chloride permeability and microstructure of portland cemen mortars incorporating nanomaterials. J Transport Res Board 2008:25:13-21.

[6] Tregger NA, Pakula ME, Shah SP. Influence of clays on the rheology of cement pastes. Cement Concrete Res 2010;40:384-91.

[7] Morsy MS, Alsayed SH, Aqel M. Effect of nano-clay on mechanical properties and microstructure of ordinary portland cement mortar. Int J Civ Environ Eng 2010;10(1):23-7.

[8] Varga G. The structure of kaolinite and metakaolinite. Építoanyag 2007;59(1):6-9.

[9] Cement concrete \& Aggregates Australia. Chloride resistance of, concrete; 2009.

[10] Abell AB, Willis KL, Lang DA. Mercury intrusion porosimetry and image analysis of cement-based materials. J Colloid Interface Sci 1999;211: 39-44.

[11] Kumara R, Bhattacharjeeb B. Study on some factors affecting the results in the use of MIP method in concrete research. Cem Concr Res 2003:33: 417-24.

[12] Rendell F, Jauberthie R, Grantham M. Deteriorated concrete. Insp Physicochem Anal. London (UK): Thomas Telford Publishing; 2002.

[13] Stanish KD, Hooton RD, Thomas MDA. Testing the chloride penetration resistance of concrete: a literature review. FHWA Contract DTFH61-97-R00022 "Prediction of Chloride Penetration in Concrete". Ontario (Canada): University of Toronto; 1997.

[14] AASHTO T277-07. Standard method of test for rapid determination of the chloride permeability of concrete. 2012.

[15] Method Nordtest. Accelerated chloride penetration and hardened concrete nordtest NT Build 443. Finland: ESPO; 1995.

[16] ASTM C1202-97. Standard test method for electrical indication of concrete's ability to resist chloride ion penetration. In: ASTM international, Philadelphia; 1997.

[17] Tang L, Nilsson LO. Rapid determination of chloride diffusivity of concrete by applying an electric field. ACI Mater J 1992;49(1):49-53.

[18] Spiesz P, Ballari MM, Brouwers HJH. RCM: a new model accounting for the non-linear chloride binding isotherm and the non-equilibrium conditions between the free- and bound-chloride concentrations. Constr Build Mater 2012;27:293-304.

[19] NTBUILD-492. NORDTEST METHOD: Concrete, mortar and cement-based repair materials: chloride migration coefficient from non-steady-state migration experiments. 1999.

[20] GB/T50082-2009. Standard for test methods of long-term performance and durability of ordinary concrete. 2009.

[21] Fan Y, Zhang S, Luan H. Influence of nano-clay addition on porosity of cement paste at early age. In: 2011 International Conference on Mechanical, Industrial, and Manufacturing Engineering (MIME 2011). Melbourne (Australia); 2011.

[22] Samet B, Mnif T, Chaabouni M. Use of a kaolinitic clay as a pozzolanic material for cements: formulation of blended cement. Cem Concr Compos 2007:29:741-9.

[23] Müller C J. Pozzolanic activity of natural clay minerals with respect to environmental geotechnics. Doctoral thesis. Swiss: University of Karlsruhe; 2005.

[24] JTGE30-2005. Test methods of cement and concrete for highway engineering. In: Chinese standard, Beijing; 2005.

[25] GB/T 50081-2002. Standard for test method of mechanical properties on ordinary concrete. In: Chinese standard; 2002. 